\title{
Salud Materno - Infantil
}

DR. LUIS GIYOVIC **

DIAGNOSTICO DE LA SITUACION DE SALUD EN EL AREA MATERNO-INFANTIL *

\section{DATOS GENERALES (1970)}

Pob. estimada del país: $\quad 9.726 .000$

" femenina: $\quad 4.980 .000$

" de mujeres en edad fértil: 2.039 .000

" niños menores 15 años: 3.822 .000

Total de la población

Materno Infantil:

Residencia Rural: $\quad 22 \%$

$5.861 .000 \quad 61,5 \%$

Residencia Urbana $78 \%$

Densidad $\quad 13,2 \times \mathrm{Km}^{2}$

Tasa de Mortalidad general: 8,6 por 1.000 habitantes.

El $50 \%$ de las defunciones acontece en el área Materno-Infantil

Sobre el $80 \%$ de estas defunciones ocurren en menores de 5 años.

Certificación médica de los fallecimientos: $81,4 \%$.

Tasa de Natalidad: 26,9 por 1.000 habitantes.

Aumento Vegetativo de la población: $1,83 \%$.

Tasa de fecundidad (nac. vivos) $119 \times 1.000$ mujeres en edad fértil.

Esperanza de vida al nacer:

Hombre: 60,86 años; mujer 66,41 años.

Viviendas urbanas: 946.080

$$
\text { rurales: } 31.800
$$

Alcantarillado: $40 \%$ de la población.

Agua Potable intradomiciliaria: $53,3 \%$ de la población urbana.

Camas hospitalarias para

Obstetricia

* Documentos de estudio.

to Ministerio de Salud. Of. de Salud Infantil.
Camas hospitalarias para

Pediatría

6.234

$75,1 \%$

Partos atendidos en establecimientos Hosp. 80,7\%

No de Consultas Gineco-Obstétricas: (SNS. 1970)

por médico:

486.839

por matrona:

1.335 .430

T o t a 1:

1.822 .269

Tasas: 0,30 consulta médica maternal anual por mujer en edad fértil, beneficiaria del SNS. y 0,82 por matrona (1).

Consulta matrona (2) anual por nacido vivo: 5,1 .

Total de consultas por nacido vivo: 7 .

No de consultas pediátricas: (S.N.S. - 1970).

(morbilidad y control de sanos).

\begin{tabular}{|c|c|}
\hline $\begin{array}{llllll}\text { por médicos: } & \ldots & \ldots & \ldots & \ldots & \ldots \\
\text { por enfermera: } & \ldots & \ldots & \ldots & \ldots\end{array}$ & $\begin{array}{r}3.550 .808 \\
896.712\end{array}$ \\
\hline a $1: \ldots$ & 4.4 \\
\hline
\end{tabular}

Tasas: 1,2 consulta médica por nin̄o menor de 15 años beneficiario del S.N.S.

0,28 consulta enfermera por niño menor de 15 años beneficiario del S.N.S.

¿DETERIOROS DE MAYOR RELEVANCIA

$$
\text { A.- NIF̃O }
$$

1._- Mortalidad:

1.1. Aún persiste alta incidencia de mortalidad en la niñez ( 0 a 4 años), no obstante su ostensible baja en los últimos 10 años. La mor-

\footnotetext{
1 Población beneficiaria se estima en um $80 \%$ det total.

Embarazo puterperio y control fecundidad.
} 
talidad de este grupo etario representa sobre $1 / 4$ de la mortalidad general. En países desarrollados menos de $1 / 10$.

El deterioro se encuentra fundamentalmente en los menores de 1 año (mortalidad infantil), por su proporcionalmente más alta tasa y porque el descenso en los últimos años no ha sido parejo.

TASAS DE MORTALIDAD INFANTIL POR 1.000 NACIDOS VIVOS Y DE NINOS DE I A 4 AÑOS POR l.000 NINOS DE ESAS EDADES

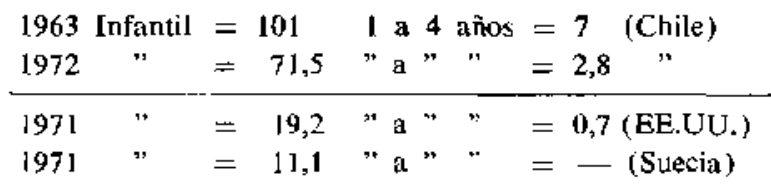

1.2. En la alta tasa de mortalidad infantil el mayor deterioro se observa en el 1.er Trimestre de la visa y muy principalmente en los menores de 28 días (mortalidad neonatal) cuyas tasas además de permanecer altas han descendido desproporcionadamente con respecto a las de mortalidad infantil; de tal manera que actualmente representa aproximadamente $1 / 2$ de la mortalidad infantil. 10 años atrás representaba apreximadamente $1 / 3$.

TASAS DE MORTALIDAD INFANTIL Y NEONATAL POR I.ONO NACIDOS VIVOS

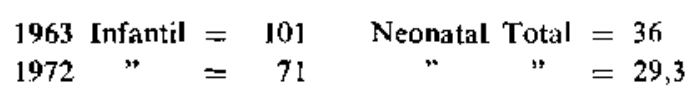

1.3. A su vez, en la alta tasa de mortalidad neonatal el deterioro se encuentra fundamentamente en los menores de 7 días (mortalidad neonatal precoz) cuya tasa, además de permanecer alta, también ha descendido desproporcionadamente; de tal manera que hoy representa $2 / 3$ de la mortalidad neonatal total.

TASAS DE MORTALIDAD POR I.ONO NACIDOS VIVOS

1963 Neonatal Total $=35,7 \quad$ Precoz $=22,7$

$1972 " \quad=29,3$ Neonatal Precoz $=19,2$
Se debe enfatizar que aproximadamente $2 / 3$ de esta mortalidad neonatal precoz ocurre en las primeras 24 horas de vida del Recién Nacido.

1.4. Esta alta tasa de mortalidad neonatal precoz unida a una también alta tasa de mortintalidad (nacidos muertos por 1.000 nacidos vivos) determina una muy alta tasa de mortalidad perinatal (suma de ambas tasas) no obstante que la de mortinatalidad no es la real, porque el registro es muy insuficiente. En encuestas realizadas a nivel local, aparecen omitidos sobre el $50 \%$ de los mortinatos.

TASAS DE MORTALIDAD NEONATAL PRECOZ MORTINATALIDAD Y MORTALIDAD PERINATAL POR I.OOO NACIDOS VIVOS

1966

1972

$\begin{array}{llll}\text { Neonatal precoz } & = & 22,9 & 19,2 \\ \text { Mortinatelidad } & = & 24,2 & 20,4 \\ \text { Perinatal } & = & 47,3 & 39,6\end{array}$

1.5. La distribución de los fallecimientos según causas, incluso los neonatales, demuestra que predominan aquéllas más evitables, propias de un bajo estrato socio-económico de la población (saneamiento ambiental, vivienda, abrigo, nutrición, etc.) y/o de una desatención de ella.

Principales causas de fallecimiento en 1970 :

(1) Menores de 1 año:

1.1. Respiratorias agudas: $32,9 \%$

1.2. Perinatales:

1.3. Diarreicas:

(2) De 1 a 4 años:

2.1. Respiratorias agudas: $31,1 \%$

2.2. Infecciosos:

$21,4 \%$

2.3. Accidentes:

La desnutrición figura como diagnóstico asociado en aprox. un $80 \%$ de las causas diarreicas e infecciosas, en un $57 \%$ de las respiratorias y en un $15 \%$ de las perinatales.

\section{2.-Morbilidad:}

2.1. Altos índices de desnutrición en todas las edades, incluyendo al Recién Nacido. No hay es- 
tadísticas confiables y anacronismo en las estimaciones.

2.2. Altos índices de prematuridad. No obstante la desuniformidad que hay en su estimación las encuestas locales dan cifras sobre $12 \%$. En EE.UU, es de sólo $7 \%$.

2.3. Desconocimiento de las cifras de morbilidad según causas. Agrupación diagnóstica de las estadísticas peca de imprecisa y los diagnósticos consignados por los médicos tíenen nomenclaturas diferentes, lo que se hace más evidente para el Recién Nacido.

No obstante ello, hay notoria prevalencia de enfermedades del aparato respiratorio y altos índices de infección intrahospitalariá en todas las edades.

2.4. No hay índices sobre morbilidad invalidante. Encuestas en universos pequeños arrojan que aproximadamente $1 / 3$ de la morbilidad del Recién Nacido es invalidante en algún grado.

\section{3.- Actividades de Organización.}

3.1. Recursos asistenciales se vuelcan prioritariamente sobre el Hospital Pediátrico postergando a los consultorios periféricos y a los sisetmas de atención pediátrica al Recién Nacido.

3.2. Hospitales Pediátricos con una organización que no jeratquiza actividades de atención quirúrgica.

3.3. Discordancia en la concepción de la organización para la mejor asistencia pediátrica de los recién nacidos, especialmente la de urgencia.

3.4. Recursos en los consultorios periféricos jnsuficientes para la atención y recuperación de la patología banal $y / o$ de los programas de protección y fomento.

3.5. Falta habilitar consultorios periféricos en poblaciones marginales ya estables. Los que hay con muy mala coordinación con Jos hospitales bases y maternidades.

3.6. Incoordinación entre los hospitales pediátri cos, consultorios y otros servicios dependientes del programa infantil que impide una información oportuna y eficiente para evitar duplicación de acciones.

3.7. No hay uniformidad de criterio en la organización y funcionamiento de salas cunas $y$ jardines infantiles. No se cumplen las dispo- siciones vigentes y la actividad se orienta más al cuidado de la salud que a proporcionar bienestar. Hay duplicación de acciones y desperdicio de horas médicas. Igual dígase de los Servicios de Bienestar en instituciones beneficiarias del Sermena.

3.8. Actividades docentes y de investigación mal jeratquizadas, mal programadas y descoordinadas con las asistenciales. Se inutilizan horas médicas y recursos de ayuda de las Universidades o de organismos internacionales.

3.9. Poca participación en los organismos o instituciones que en alguna forma se orientan a a la búsqueda del bienestar familiar, o que les corresponde dictar códigos o leyes que se relacionan con la actividad materno infantil.

\section{4.- Actividades normativas y programáticas.}

4. l. No hay normas comunes de organización y funcionamiento de los hospitales pediátricos, entre ellos y en relación a otros servicios, secciones, unidades, consultorios de especialidades o periféricos, dependientes de un programa pediátrico.

4.2. No hay promoción para la dedicación de los profesionales a la especialidad pediátrica $y / 0$ a la especialización en las áreas más deterioradas como: cirugía, neomatología, radiología y anatomía patológica infantiles.

4.3. Poca preocupación por el perfeccionamiento del personal auxiliar en la problemática de la especialidad.

4.4. No hay normas para el uso de fichas clínicas de evaluación o información similares. El procesamiento estadístico de la morbilidad con diversas nomenclaturas se hace imposible.

4.5. Control del niño sano de baja cobertura y poco eficiente (sólo medidas antropométricas $c$ instrucciones dietéticas).

4.6. Programa de alimentación suplementaria cuestionable y no evaluado.

4.7. Programa de vacunaciones de cobertura no suficiente.

4.8. Control del niño escolar esporádico e insuficiente.

4.9. No se ha delimitado el campo de acción sobre el adolescente.

4.10. No hay programas adecuados para la rehabilitación física y mental del niño. 


\section{B. MADRE}

1.--Mortalidad:

1.1. Ha disminuido notoriamente pero aún persisten tasas regionales relativamente elevadas. Las defunciones maternas son en su mayoría evitables. Causas predominantes son: toxemía, hemortagia y sepsis. Las defunciones por sepsis son en su mayoría consecuencia de abortos sépticos provocados. La causal aborto representa $1 / 3$ de la mortalidad materna.

Tasas de mortalidad materna y por aborto por 1.000 Nacidos Vivos

\begin{tabular}{|c|c|}
\hline $\begin{array}{l}1963 \text { Totales }=2,6 \\
1972 \text { Totales }=1,6\end{array}$ & $\begin{array}{l}\text { Por aborto }=1 \\
\text { Por aborto }=0,5\end{array}$ \\
\hline
\end{tabular}

\section{2.--Morbilidad:}

2.1. La alta incidencia de toxemia, hemorragia y sepsis repercute en las altas cifras de mortalidad perinatal.

2.2. La ocupación de camas para atender esta morbilidad y el aborto disminuye el recurso cama para puerperio, lo que obliga al alta precoz. Variada patología que puede acontecer en el neonato (enf. hemorrágica - ictericia, sepsis, etc.) en los primeros días de la vida, escapa al control pediátrico. Esta situación contribuye a aumentar la mortalidad neonatal y las secuelas invalidantes de la morbilidad.

2.3. Alta incidencia de complicaciones del embarazo (procidencia del cordon, ruptura uterina, sufrimiento fetal, etc.) y de parto prematuro que repercuten en aumentar la morbimortalidad perinatal y secuelas invalidantes en el recién nacido. Ello se deriva en gran parte de mal control de la embarazada y concurrencia tardía a la maternidad.

\section{3.- Actividades de organización.}

3.1. Normas de organización y funcionamiento de las Maternidades no dan la jerarquía que se merecen los sistemas de atención pediátricas, especialmente la urgencia.

3.2. Mala coordinación de las Maternidades con los Hospitales pediátricos y consultorios periféricos para el denuncio del nacimiento $\mathrm{y} / \mathrm{o}$ de la patología acontecida; lo que retarda el control oportuno y/o efectivo del recién nacido $y$ duplica acciones.
3.3. Aumento desproporcionado entre el porcentaje de gestantes que tienen sus partos en establecimientos Hospitalarios y el número de camas obstétricas ha determinado un deterioro de la disponibilidad, que obliga al alta precoz y a la ocupación de "cama caliente" y hasta de 2 por cama.

3.4. Convenios con clínicas particulares para la asistencia de Ias puérperas obliga a traslados en condiciones sub humanas e impide el control del recién nacido; lo que aumenta los índices de morbilidades y mortalidad neonatal.

3.5. La notoria insuficiencia de recursos y tecnología para la pesquisa precoz del sufrimiento fetal aumenta la morbilidad y mortalidad perinatal y fundamentalmente eleva los indices de morbilidad invalidante.

3.6. Falta de control y educación para el uso y poce de permisos maternales determina un bajo rendimiento de este recurso. El agotamiento físico de la embarazada aumenta la morbilidad perinatal y los índices de inmadurez. La distracción psíquica de la puérpera inhibe la producción láctea.

\section{4.- Actividades normativas y programáticas.}

4.1. Controi maternal muy insuficiente. Tasa aproximada en el país es de 1,12 consulta anual por mujer en edad fértil de la cual śblo $1 / 4$ es realizada por médico.

4.2. La programación de estos controles adolece de las siguientes principales deficiencias: a) mala coordinación con el laboratorio e insuficiencia de exámenes; b) actividad no integrada con las de control de fertilidad o pesquisa del cáncer cérvico uterino; c) inasistencia a los controles no se investiga; d) mala coordinación con la Maternidad donde ocurrirá el parto que impide conocer oportunamente el diagnóstico del embarazo, o el resultado de exámenes; e) poca preocupación por facilitar el traslado oportuno de la parturienta que esteriliza todo esfuerzo, etc.

4.3. Programa de Planificación Familiat sólo mantiene preocupación por la regulacion de la fertilidad minimizando todas las otras acciones que una concepción adecuada exige. La baja tasa de cobertura de esta regulación para las mujeres en edad fértil de todo el país tampoco justifica los esfuerzos. Iguales re. cursos humanos bien programados podrían aumentar notiriamente el rendimiento y cubrir otras acciones de bienestar familiar. 
4.4. No hay un programa adecuado de fomento de la lactancia natural. Está permanentemente deteriorado por: a) entrega indiscriminada de leche a tempranas edades; b) bajo subsidio maternal; c) incumplimiento de la ley de salas cunas; d) mal uso del reposo post natal, etc.

\section{RESUMEN}

En el area Injantil, que comprende sobre el 60\% de la población del pais, puede evidenciarse:

I.- Altos índices de morbimortalidad infantil con mayor ocurrencia a menor edad y muy fundamentalmente en el periodo perinatal.

2.-Predominio de la desnutrición como diagnóstico causal o asociado.

3.- Relación estrecha del curso del embarazo y parto con la probabilidad de sobrevida y estado de salud del recién nacido.

4.- Alta incidencia de morbilidad invalidante para el recién nacido y de muerte materna por aborto.

5.- Establecimientos asistenciales del área materno infantil con una organización y funcionamiento que no considera esta realidad y que coadyuva a:

1) Limitación de la expectativa de vida al nacer;

2) Contribucion importante al deterioro socio-económico de la Nación.

\section{CRITICAS A LA ACTUAL ESTRUCTURA NORMATIVA MATERNO INFANTIL}

Las normas de salud materno infantiles ema. naron hasta la fecha de la sección Materno Infantil del Subdepartamento de Fomento de la Salud, que es a su vez dependiente del Departamento Técnico de al Dirección General del Servicio Nacional de Salud; lo que acarrea los siguientes inconvenientes:

1) Por emanar del Subdepartamento de FoFomento de la Salud pareciera que las normas a dictar sólo debieran ser las inherentes a las acciones de Fomento, con exclusión de las de Protección o Recuperación de la Salud; pero cómo en la práctica también inciden en estas otras dos acciones esta sección tiene una autoridad normativa indiscutible en todo lo que điga relación con las acciones de Protección y Recuperación de Ia salud materno-infantil, que se realizan en cumplimiento de normas integradas dictadas en un contex to único.

2) Siendo así no se ve la razón para que esta sección se encuentre dependiente de la SubeJfatura de Fomento de la Salud y, no de la de
Protección o Recuperación, ni tampoco para que necesariamente tenga que ser dependiente de alguna de ellas, o el por qué en cada una no pudiera haber una Sección Materno Infantil.

3) Aparece en cambio claramente visible que la práctica ha demosteado la necesidad de una estructura que tenga autoridad para dictar con un mismo criterio las normas más adecuadas, de manera tal que en el nivel ejecutivo último se realicen acciones coordinadas de Protección Fomento y Recuperación a la vez; evitando con ello situaciones conflictivas o dispendio de recursos que podría ocurrir si éstas actividades estuviesen sometidas a diversos criterios normativos.

4) En consecuencia si en la práctica se ba entregado la actividad normativa materno infantil a esta sección, desde la que se están dictando normas integradas de protección fomento $y$ recuperación a la vez bajo un mismo criterio, es indudable que en el organigrama del SNS debería aparecer en una posición que permitiera que si estas actividades permanecen individualizadas se vean dependientes, y no una de ellas mandante del Programa Materno infantil como actualmente ocurre.

5) Igualmente si la actividad salud Materno Infantil es hegemónica con respecto a las actividades de Fomento Protección y Recuperación, junto con darle una ubicación por sobre ellas debería habérsele otorgado una jerarquía de más alto nivel, y no mantenida minimizada en una sección tan dependiente.

6) En todo caso cualquiera hubiese sido la ubicación de la sección materno infantil dentro del organigrama del Servicio Nacional de Salud no aparece lógico que, aun cuando este sea el Servicio prestario de Salud mayoritario, sea el que esté dictando normas con respecto a los otros por pequeños que fueren.

7) Tampoco aparece lógico que los convenios de ayuda nacionales o extranjeros para mejorar el desarrollo o la atención de la Madre o el Niño sean entregados para su aplicación a sólo en uno de los Servicios de Salud, privando de eventuales coberturas a beneficiarios de otros muchas veces de más bajos ingresos, aun cuando gocen de la categoría de empleados.

FUNDAMENTOS DE LA NECESIDAD DE UNA ESTRUCTURA FUNCIONAL NORMATIVA UNICA PARA EL AREA MATERNO INFANTIL.

1.- Si se acepta como política un conjunto de proposiciones inteligentes que tengan como objetivo solucionar problemas definidos, las 
que en este cometido se dicten en cl área de la Salud Materno Infantil, para que sean coherentes, deberán ser consecuentes con algunas connotaciones que aparecen claramente visibles en estat enunciación de problemas de mayor jerarquía en nuestra país, cuales son:

1.1. Hay una estrecha relación entre la atención del embarazo $y / o$ del parto y el menor riesgo de detcrioro de la salud del recién nacido.

1.2. Una proporción no despreciable de la patología neonatal es derivada de morbilidad materna.

1.3. Las acciones de recuperación, fomento y protección aparecen como un todo inseparable en las consecuencias o resolución de la problemática expuesta.

1.4. Los programas de atención a la madre y al niño tienen implicaciones mutuas que requieren una acción unitaria y coordinada en el nivel normativo y ejecutivo último.

\section{2.- En consecuencia:}

2.1. Como buena parte de las actividades que se proponen necesariamente deberán comprender en conjunto al binomio madre y niño, se hace necesario y aconsejable mantener en el nivel normativo un grado de integración que polarice esfuer7os hacja el objetivo común, y de dirección que no se aparate de este esquema conceptual.

2.2. Si sc considera que la mayor parte de las acciones necesarias requieren una atención integrada con actividades de recuperación, fomento y protección de la salud, que finalmente en el nivel ejecutivo último quedan bajo la responsabilidad o supervisión de un mismo profesional, se hace a su vez aconsejable que las normas mantengan también este esquema conceptual de integración de eslas actividades, bajo la dirección de un mismo criterio. Esta proposición no se contrapone en absoluto con cualquiera dispersión de ellas que pueda hacerse aconsejable en el nivel ejecutivo intermedio, para una mejor distribución de recursos materiales o humanos, o pari una más expedita programación de algunas claramente individualizables.
3.- Correspondiéndole al Ministerio de Salud dictar todas las normas a que deben ceñirse los organismos ejecutivos que otorgan servicios de Salud, es indudable que deberá disponer de una estructura funcional donde en un mismo nivel se sitúe la actividad materno infantil y las otras de objetivo común que aparezcan necesarias para que el sistema nacional de servicios de salud cumpla sus propósitos.

(Ver organigrama).

En un nivel paralelo deberá también situarse la estructura que le corresponda adecuar y distribuir los recursos del sistema. La centralización en el nivel ministerial de todos los recursos obtenidos a través de convenios con organismos nacionales o internacionales aparece indispensable para dictar normas de utilización comunes e igualitarias entre todos los Servicios del Sistema de Salud.

4.-- Sólo una vez producido el encuentro y acuerdo entre cada una de las estructuras de actividades técnicas y la de utilización de recursos, se está en condiciones de planificar, estableciendo metas con evaluacióni y control permanente; lo que exige otra estructura funcional de asesoria en planificación y evaluación, ubicada también en el nivel normativo.

\section{VENTAJAS DE LA NUEVA POLITICA DE SALUD MATERNO INFANTIL PROPUESTA}

1) Se separan claramente los ámbitos normativos y ejecutivos, entregando los primeros al Ministerio y manteniendo los ejecutivos en los organismos que prestan Servicios de Salud.

2) Se soluciona el impasse que significaba mantener el poder normativo en sólo uno de los organismos que prestan servicios de salud en desmedro de los otros. No es igual que las normas las dicte el Ministerio a que las dicte sólo uno de los Servicios interesados.

3) Se le da a la actividad normativa materno infantil la jerarquía que se merece, consecuente con la política de prioridades que se ha fijado el Supremo Gobierno para otorgar salud a través de este Sistema Nacional de Salud; dependiente en lo normativo del Ministerio de la Salud cuya tuición sobre todos los organismos que prestan salud sean estatales o privados queda claramente definida.

4) Se deja definitivamente establecido que las normas deben ser únicas, integradas y emanadas de un mismo criterio, para todo lo que diga relación con acciones de recuperación, fomento o protección de la salud del niño o de 
Para hacerlo comprensible we muestra el organigr'tma actual del SNS.

DIREC. DE LOGISTICA

En recuadto negro aparecen las estructuras normativas ministeriales propuestas.

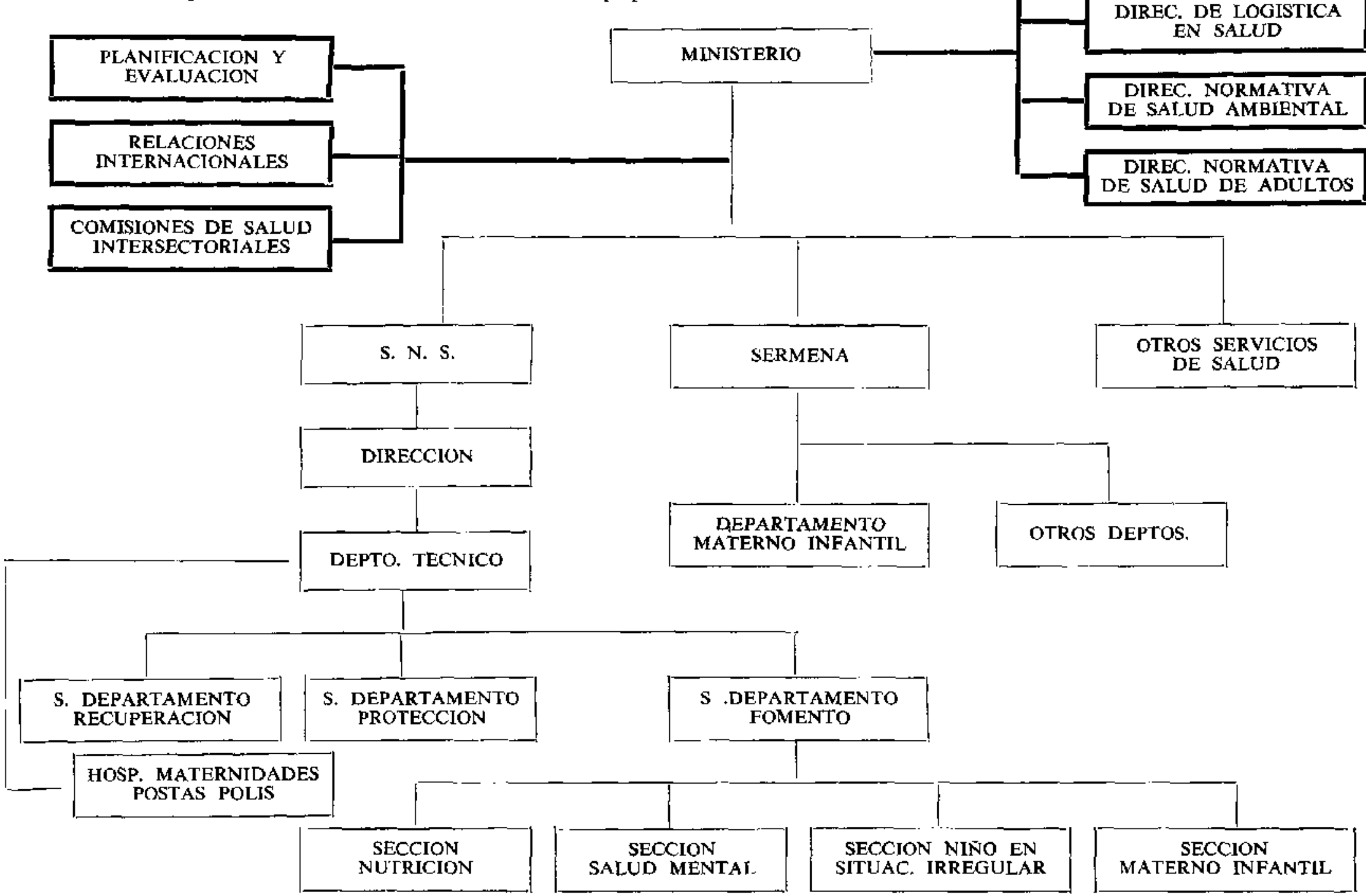


la embarazada. Esta división artificial para cada una de estas actividades no agilizaba las acciones y hacía engorroso y conflictivo su cumplimiento al nivel ejecutivo último.

5) Se entregan las normas de distribución de recursos, y muy especialmentee de los obtenidos a través de convenios de ayuda internacionales, a la cabeza normativa de este Sistemit Nacional de Salud, de tal mödo que puedan beneficiarse todos y no sólo uno de los servicios que prestan salud.

\section{OBJETIVOS DE UNA CENTRALIZACION NORMATIVA EN EL AREA MATERNO INFANTIL}

Vistos:

- Los propósitos de atención preferente a la madre y al niño, declarados por el Supremo Gobierno y señalados en la Política de Salud que preconiza el Ministerio de Salud.

- El Plan cuadripartito de operaciones para un Programa de Extensión de Servicios de Salud Materno Infantil y de Bienestar Familiar en Chile, PESMIB), suscrito por el Gobierno de la República de Chile, la Organización Panamericana de la Salud (OPS) el Fondo de las Naciones Unidas para la Infancia (UNICEF), las Naciones Unidas (NU) y el fondo de las Naciones Unidas para actividades de población (FNUAP), vigente desde el 27 de Diciembre de 1972 y hasta el 31 de Diciembre de 1976.

- Los problemas de mayor relevancia que afectan Ios programas de salud materno infantil senalados en el documento adjunto y las metas propuestas en el Convenio vigente.

Me permito proponer una centralización normativa para el área Materno-Infantil con los siguientes objetivos que permitirán cumplir sus metas u otras:

1.- Racionalizar los recursos materiales y humanos en los Hospitales - Consultorios y Maternidades dependientes del Servicio Nacional de Salud con los siguientes objetivos inherentes a esta problemática.

1.1. Que en los Hospitales Generales o en las Maternidades haya normas de organización y funcionamiento que permitan coordinar las acciones entre los Servicios de Obstetricia y los sistemas de asisten cia Pediátrica, de tal manera que la atención prestada sea eficiente, oportuna c igualitaria para la madre y el hijo, dándole proiridad a la problemática perinatal.
1.2. Que en los Hospitales Generales o Servicios de Pediatría se dé atención preferencial a la consulta ambulatoria de las diversas especialidades y de la urgencia; y las camas o cunas destinadas preferencialmente a la hospitalizacion de casos agudos o de solución quirúrgica.

1.3. Que en los Consultorios periféricos se resista la presión asistencial para recuperación de la salud deteriorada por patología banal y se dé cumplimiento a las siguientes acciones médicas de protección y fomento;

1.3.1. Control periódico del niño con un enfoque particular para los diversos grupos etarios; lactante, preescolar, escolar y adolescente.

1.3.2. Control periódico obligatorio de la embarazada y atención de su morbilidad con práctica de exámenes de laboratorio de rutina.

1.3.3. Cumplimiento obligatorio de los programas de vacunación pre determinados.

13.4. Planificación Familiar en la real extensión de su significado de bienestar familiar, que considere entre otras problemáticas las de Fertilidad - Esterili-dad - Aborto Inducido - Alimentación Natural - Reposos Preventivos - Rehabilitación y Control del niño parcialmente invalidado - Educacion en Salud - Enfermedades Venéreas - Conducta sexual - Paternidad Responsable - Control del Cáncer Cérvico, Uterino, etc.

El control de la fecundidad debe hacerse extensivo a toda la población beneficiaria de Servicios de Salud, y no sólo a la del Servicio Nacional de Salud.

1.3.5. Otorgar suplementación alimentaria al niño y a la embarazada con la calidad y cantidad que determinen programas adecuadamente planificados $y$ evaluados.

1.3.6. Ofrecer medios de movilización oportunos desde los consultorios a los Hospitales o Maternidades para el traslado de enfermos o muestras para exámenes de Latbotario.

1.4. Propender a instalar en las poblaciones urbanas ya estables, o campamentos que no los hubiere, locales para habilitar 
Consultorios con la pacticipación de los propios pobladores, tanto para la construcción como para la formación posterior de líderes en salud que puedan colaborar y cumplir algunas de las actividades anteriormente enunciadas.

1.5. Que todos estos establecimientos de saIud realicen acciones estrechamente coordinadas con información mutua permanente y oportuna a través de registros y papeletas de comunicacion tendiendo a:

1.5.1. Uniformar las nomenclaturas $y$ aún los criterios diagnósticos.

1.5.2. Estandarizar las fichas clínicas, las de evaluación de daños, las de información, las de estadística, etc., haciéndolas en to posible precodificadas.

1.5.3. Establecer nuevas tablas de valoración de la desnutrición infantil que exijan incrementos periódicos en vez de promedios totales para cada edad.

1.5.4. Controlar y exigir el cumplimiento de las actividades obligatorias.

1.5.5. Obtener índices fidedignos de mortalidad y de morbilidad, especialmente de aquellas invalidantes.

2. - Impartir normas para que se realicen simiJares adecuaciones que en los establecimientos del S.N.S. en los otros dependientes del Ministerio de Salud o de otros Ministerios, en lo que no se contraponga abiertamente con sus propias normas de utilización de sus recursos.

3.- Velar por el cumplimiento de las disposicio. nes sanitarias que dicen relación con la habilitación de salas cunas y jardines infantiles con el objetivo de fomentar la alimentación natural y procurar confort y bienestar a los niños, excepto lo que diga relación con problemas de salud que son de otra competencia.

4.- Proponer a las autoridades o asesorar a participar en la formulación o reestudio de leyes que digan relación con el niño o la embarazada, en procura de obtener el mayor beneficio, dándole prioridad a los de adopción, subsidios y reposo preventivo.

5.- Promover las siguientes otras acciones que vayan indirectamente en bencficio del niño $y$ de la embarazada:
5.1. Formación de profesionales universitarios y de personal auxiliar de colaboración médica especializados en Ia atención del Recién Nacido.

5.2. Estimular la dedicación médica a especialidades como anatomía patologica, radiológica y cirugía infantiles.

5.3. Promover el perfeccionamiento científico y la supervisión permanente del equipo de salud que se desempeña en el área materno infantil.

5.4. Habilitación đe Centros de investigación y docencia dotados de alta tecnología para el estudio de la problemática materno infantil.

5.5. Obtener, orientar o consolidar aportes económicos del país o del extranjero entregados para definidas acciones de salud que competan al área materno infantil.

5.6. Propender a que los Convenios de ayuda con OPS/OMS, UNTCEF, APRO. FA U otros destinados a la protección de la familia, el niño o la embarazada, sólo puedan operar o funcionar bajo la dirección normativa del Ministerio de Salud.

\section{METAS}

Aún cuando no se ha definido la distribución de los recursos de salud, el curnplimiento de los objetivos señalados en este Programa permite establecer algunas de las metas que se señalan como un logro tentativo para el decenio que está transcurriendo, cuales son:

1.- Disminuir la mortalidad infantil en un $40 \%$.

2.-Disminuir la mortalidad del niño de 1 a 4 años en un $20 \%$.

3.-Disminuir la mortalidad neonatal en un $30 \%$.

4.-Disminuir la mortalidad materna en un $30 \%$.

5.- Disminuir la mortalidad por aborto en un $50 \%$.

6.-Disminuir la mortalidad por desnutrición en un $30 \%$.

7.-Aumentar los controles de niño sano en un $40 \%$.

8.- Aumentar los controles de la embarazada en un $40 \%$. 
9.- Aumentar las vacunaciones en un $15 \%$.

10.- Asegurar la cobertura de suministro de alimentación suplementaria al $80 \%$ de las embarazadas y niños menores de 5 años.

11.- Extender la cobertura de regulación de fertilidad al $30 \%$ de las mujeres en edad férti lde toda la población del país .

En resumen, aumentar la expectativa de vida al nacer y mejorar el nivel socio económico de la Nación.

PLAN CUADRIPARTITO DE OPERACIONES PARA UN PROGRAMA DE EXTENSION DE SERVICIOS DE SALUD MATERNO-INFANTIL Y DE BIENESTAR FAMILIAR

\section{PARTICIPANTES:}

Agencia Cooperadora:

Gobierno de Chile.

Agencias ejecutivas:

1. Organización Mundial de la Salud OMS).

2. Organización Panamericana de la Salud OPS).

3. Fondos de las Naciones Unidas para la Infancia (Unicef).

4. Naciones Unidas (NU) que proporciona el Fondo de las Naciones Unidas para Actividades de Población (UNPFA) como agencia financiadora.

Financiamientos:

UNPFA: EUA. $\$ 3.198 .750$.

Distribuidos año a año según cuadro adjunto.
Fecha del convenio: 27 de Diciembre de 1972. Fecha iniciación: $1^{19}$ de Enero de 1973.

Período de duración: 1973-1976.

Dirección y ejecución:

1.- Un Director del proyecto que designará el Gobierno.

2.- El Representante Residente del Programa de desarrollo de las Naciones Unidas.

3.- Un Consejo Ejecutivo compuesto por los Jefes del Sub-Departamento de Fomento de la Salud y de la Sección Materno Infantil del Servicio Nacional de Salud y el equipo multiprofesional con tres médicos, 2 enfermeras, 4 matronas, una educadora de párvulos y tres secretarias que laboran en la Sección Materno Infantil.

4.-Personal de otras dependencias que sea necesario incorporar.

5.-Un Director de Investigación y Evaluación del proyecto con una unidad compuesta por el siguiente equipo: 1 Estadístico analista y otro de computación, 1 enfermera, 1 matrona, 1 asistente social, 3 perforistas, 2 secretarias y 1 sociólogo, designados por el Gobierno. materno infantil, designados por el consejo ejecutivo.

6.- Los Jefes de Zona, Area, Servicios, programas, etc., que digan relación con la atención materno infantil, designa dos por el consejo ejecutivo.

\begin{tabular}{|c|c|c|c|c|c|}
\hline Rubro & 1973 & 1974 & 1975 & 1976 & $\begin{array}{c}\text { TOTAL } \\
\text { \$EUA } \\
\end{array}$ \\
\hline $\begin{array}{l}\text { 1. Asistencia Téc. } \\
\text { 2. Materiales y Eq. }\end{array}$ & 20.000 & 20.000 & 20.000 & 20.000 & 80.000 \\
\hline $\begin{array}{l}\text { 2. Habilitación } \\
\text { Hat. }\end{array}$ & 197.000 & 142.100 & 131.950 & 131.950 & 603.000 \\
\hline Transporte & 106.400 & 71.400 & 71.400 & 53.900 & 303.100 \\
\hline Anticonceptiv. & 143.550 & 232.700 & 282.100 & 351.500 & 1.009 .850 \\
\hline 3. Escuelas Profesionales & & & & & \\
\hline $\begin{array}{l}\text { Materiales } \\
\text { Transporte }\end{array}$ & 35.000 & 34.200 & 34.200 & 34.000 & 137.400 \\
\hline $\begin{array}{l}\text { Transporte } \\
\text { 4. Adiestramitnto en el }\end{array}$ & 30.000 & 30.000 & - & - & 60.000 \\
\hline $\begin{array}{l}\text { 4. Adiestramicnto en el } \\
\text { extranjero } \\
\text { 5. Adiestramiento en el }\end{array}$ & 47.000 & 45.000 & 45.000 & 45.000 & 182.000 \\
\hline país & 45.000 & 45.000 & 45.000 & 45.000 & 180.000 \\
\hline $\begin{array}{l}\text { 6. Investigación } \\
\text { 7. Educ. integral }\end{array}$ & 72.600 & 83.600 & 83.600 & 83.600 & 323.400 \\
\hline de la familia & 50.000 & 50.000 & 50.000 & 50.000 & 200.000 \\
\hline 8. Costos administrativos & 30.000 & 30.000 & 30.000 & 30.000 & 120.000 \\
\hline TOTAL & 776.550 & 784.000 & 793.250 & 844.950 & 3.198 .750 \\
\hline
\end{tabular}


7. - Todas las personas mencionadas y otras que pudieren aparecer necesarias pueden ser contratadas o recibir subvenciones o subsidios por horas extraordinarias de trabajo.

8. - Los directores el Representante y los de cada una de las Agencias ejecutivas integran un Consejo Consultivo y otro Técnico-Administrativo.

\section{Obligación:}

1.- Evaluación semestral indicando gestiones o modificaciones en los planes de trabajo.

2.- Inventario anual del equipo provisto que permanece como patrimonio del proyecto.

3. - Revisión del proyecto a los 18 meses de iniciado para determinar cambios o modificaciones.

4.- El plan de operaciones podrá ser modificado, terminado o prolongado en cualquier momento por acuerdo de las partes.

Propósitos generales del proyecto:

1.- Extensión de los Servicios de Salud Materno Infantil y de Bienestar familiar.

2.-Disminución de la morbimortalidad materna, fetal, perinatal y del niño.

3.- Promoción de la educación en salud y de la paternidad responsable.

\section{Actividades programadas:}

\section{1.- Protección y Fomento:}

1.1. Aumentar progresivamente el número de controles del niño y de la embarazada hasta lograr una cobertura de $70 \%$.

1.2. Aumentar progresivamente el número de vacunaciones hasta lograr una cobertura de $90 \%$.

1.3. Aumentar progresivamente el número de visitas domiciliarias.

1.4. Aumentar progresivamente el suministro de leche a lactantes, preescolares y embarazadas hasta lograr una cobertura de $90 \%$.

1.5. Aumentar progresivamente la atepción institucional del parto hasta una cobertura de $80 \%$.

1.6. Ponderar el riesgo obstétrico perinatal para adecuar recursos.

1.7. Detección precoz del cáncer cérvico uterino.
1.8. Aumentar progresivamente la regulación de la fecundidad hasta lograr una cobertura de $30 \%$ de la población del país; distribuyendo anticonceptivos también en las áreas que el proyecto no considera.

1.9. Promover la participación y educación en la comunidad.

\section{2.- Recuperación:}

2.1. Redistribución de los recursos humanos.

2.2. Aumentar la disponibilidad de horas profesionales y auxiliares en un $30 \%$.

2.3. Aumentar la cobertura médica de las consultas por morbilidad.

2.4. Mejorar la atención materno infantil.

2.5. Mejorar la promoción de los profesionales que cubren el área materno infantil y la calidad de la atención de salud materno infantil.

2.5.1. Modificar curriculum de estudios de Escuelas profesionales.

2.5.2. Otorgar becas de adiestramiento en el extranjero.

2.5.2.1. 17 becas por año de 4 meses de duración (cortas).

1 beca por año de 10 meses de duración (Académicas).

4-3-3-3 becas en cada año consecutivo de 4 meses de duración (sólo para ejecutivos.

2.5.3. Otorgar material didáctico a Escuelas de Obstetricia, Enfermería, Tecnología, Nutrición y Medicina.

2.5.3.1. Módulos didácticos.

2.5.3.2. Libros y textos.

2.5.3.3. Material Audiovisual.

2.5.3.4. Documentos fílmicos de Salud Materno Infantil y Planificación Familiar.

2.5.4. Creer un Centro Nacional para proporcionar el material audiovisual.

2.5.5. Promover vehículos (mantenimiento de cargo del Gobierno).

2.5.5.1. 5 Buses en 1973 y 5 en 1974 para Escuelas de Enfermería y Obstetricia. 28-18-19 "Land Rover 88" en cada año consecutivo para el cumplimiento de actividades docentes.

2.6. Proveer equipos y medicamentos en base a un diagnóstico previo de necesidades. 
2.7. Equipar 4 Centros Regionales (OsornoPuerto Montt-Temuco y Valdivia) especializados en asistencia $y$ adiestramiento en salud materno infantil.

\section{RECTIFICACIONES RECOMENDABILES}

En el proyecto en referencia se hace necesario incorporar modificaciones adecuadas a la realidad de la actual política de salud que impulsa el Supremo Gobierno cuales serían:

1.-Dejar claramente establecido que como el ambito normativo de la salud reside actualmente en este Ministerio a él corresponde dictar las normas adecuadas para el cumplimiento, evaluación y supervisión del Proyecto. El Servicio Nacional de Salud y los Servicios Estatales de Salud, dependientes o no de este Ministerio, que eventualmente pudieren beneficiarse del proyecto pasan a ser los organismos ejecutivos de las normas que se dicten.

Ello exige que los nombramientos de las personas a catgo de la Dirección y de la Evaluación del Proyecto recaigan en quienes no sean a su vez los responsables de su ejecución.

2.- Suprimir todas aquellas alusiones que se bacen al Seryicio Unico de Salud y al Ministerio de la Familia; metas preconizadas por el anterior Gobierno que indujeron a proponer la responsabilidad del proyecto al Servicio Nacional de Salud, que tácitamente aparecía como la cabeza visible normativa y operativa del futuro Servicio Unico.

3.- Propender a incluir dentro de las áreas programáticas del proyecto a los beneficiarios de los otros Servicios Estatales que otorgan salud; especialmente en lo que se refiere a control de fecundidad, cuyo alto costo actual margina a un grupo social de clase media muy desvalido. Si así no se decide indudablemente ocurrirá un aumento de la mortalidad por abortos, con la consecuencia de morbimortalidad materno infantil que le acompaña.

4.- También debe reestudiarse las áreas programáticas a incorporar en los siguientes años, de tal manera que no queden marginados algunos centros conflictivos y de alta concentración obrero de mineros como TocopillaCalama-Coquimbo-Los Andes-Rancagua-Coronel y el área Oriente de Santiago, la que además ba incrementado explosivamente st población marginal.
5.- Mientras el plan séa modificado, de acuerdo a estas sugerencias u otras que pudieren aparecer necesarios, este Ministerio según las propias disposiciones del proyecto está en su derecho para designar al Director del proyecto y al de la Unidad de Investigación y evaluación, sin perjuicio de confirmar a todos los funcionarios del Subdepartamento de Fomento y Departamento Técnico del Servicio Nacional de Salud que en su calidad de tales les corresponda integrar el Consejo Ejecutivo y la Unidad de investigación y evaluación respectivamente. Estos nombramientos permitirían desde ya que el proyecto sea dirigido y evaluado por el Ministerio de Salud y específicamente desde el organismo que para normar en Salud Materno Infantil se acuerde crear.

EL CONTROL DE LA FECUNDIDAD Y EL DESARROLLO SOCIO-ECONOMICO

Chile es un país privilegiadamente sin grandes problemas demográficos por su actual tasa de crecimiento vegetativo de la población, la que en cambio para otros países en desarrollo aparece como un desafío y una seria amenaza de subsistencia. Muchos son los factores que pueden estar limitando nuestros índices de fecundidad pero, a juzgar por la disminución paralela tanto de la mortalidad general como de la natalidad, no cabe duda que el tal vez más determinante lo constituye la regulación de la fecundidad que cumplen Ias familias, espontáneamente o con asistencia médica, en su mayoría sin respaldo económico oficial ni como fruto de actividades programáticas concebidas con ese fin por servicios médicos estatales. Va en apoyo de esta afirmación la circunstancia que estando un $43 \%$ de la población femenina del país en eđad fértil aproximadamente un $10 \%$ de elia, y sólo de las que son beneficiarias del SNS., están sometidas a un plan de control; y aún menos si se considera que este porcentaje se ha determinado más en base al número de consultas que al de personas año regularmente atendidas. Si se ha llegado a estos resultados con esta cobertura tan baja, que deja marginadas casi a un $90 \%$ de las mujeres beneficiarias del SNS, en edad fértil, y al $30 \%$ restante de la 
población femenina del país que por la circunstancia de no ser beneficiarias del SNS se les mantiene excluídas, no se puede negar que en este aspecto más ha hecho la concientización de la promoción anticoneeptiva, o el convenciniento de su necesidad ante la limitación de los ingresos de la familia, que las actividades programáticas de regulación de la fertilidad per se. Si lo hacen con las metódicas que estos programas inducen o a través de la práctica del aborto es una interrogante que debe investigarse, sobre todo si se considera que aún tenemos altos índices de mortalidad materna en los que la causal aborto representa $1 / 3$ de ella.

La pregunta que surge es si hay rangos de variación en el número de hijos de cada familia de tal índole que entre extremos muy pronunciados se obtenga un promedio favorable, $y$ si aquellos con menos hijos tienen un mayor nivel socio-económico y cultural que les permitió tomar conciencia del problema. Si así fuere significaría que estamos incrementando nuestra población con niños que por haber sido concebidos sin responsabilidad, y seguramente crecidos y desarrollados en ambientes más hostiles, pudieran tener daños biológicos irrecuperables que cuando adultos les in pedirá cumplir cabalmente el rol laboral que Ia comunidad les exigirá y para ello quiera requerirlos, y muy fundamentalmente que la parte de población más descubierta por los programas ofjciales de regulación de la fecundidad bien pudiera estar acudiendo a la práctica del abotto, y contribuyendo por su alta probabilidad de muertc a restarle a la comunidad no sólo los niños, que debemos suponer con mjeores espectativas, sino además las madres más dispuestas a aceptar responsablemente una regulación de su fecundidad. Hago abstracción de las trasgresiones éticas a que además se ven compelidas al tener que decidir la muerte de un ser con vida que sólo está curapliendo su ciclo biológico de crecimiento y desarrollo intrauterino para nacer a la vida extrauterina.

Todo hace entonces aconsejable dar siempre a los programas de control de la fecundidad un carácter nacional, no excluyente de determinados grupos sociales, sobre todo si se considera que el esquema conceptual para su promoción ha sido evitar el aborto. Limitarlo sólo a algunos beneficiarios significa dejar marginados a otros con iguales derechos, y no cumplir con sus objetivos. Los costos de los métodos anticonceptivos, y de las prestaciones de servicios que se involucran, lo hacen imperativo cuando bay una política declarada contra el aborto ilegal.

Por otro lado si nuestra tasa de $1,8 \%$ anual de crecimiento vegetativo se mantuviese con ese ritmo sólo al cabo de casi 40 años vendríamos a duplicar el número de habitantes de la nación, en circunstancias que al decir de los economistas con un mejor aprovechamiento de nuestras riquezas básicas y mayor expansión del potencial agropecuario-minero e industrial del país, estaríamos en condiciones de alimentar una población al menos 3 veces superior. Lo que no se ha dicho es que un requisito ineludible para alcanzar esta meta debe ser aumentar los brazos y las mentes abocadas a estas tareas, vale decir la población laboral. Una conclusión simplista y no bien meditada induciria hacerlo con una política de indiscriminado y acelerado incremento poblacional, olvidándose de todos los problemas biológicos, psicoemocionales, econónicos, culturales, sociales y de toda índole que se acarrea cuando en una explosión demográfica se sobrepasa el equilibrio que debe buscarse con un incremento paralelo de la producción y de los bienes y servicios. Si para una política de expansión socio-económica necesitáremos mayor mano de obra debemos estudiar previamente si con este ritmo o aún menor incremento de la población, y una racionalización de los recursos materiales y humanos, inteligentemente concebida y mejor ejecutada la pudiésemos cumplir. Debemos sí considerar que los países desarrollados para lograrlo debieron previamente incrementar en una mayor proporción su población, y aún la continúan aumentando en base a emigración seleccionada, y nosotros que no podemos garantizar hasta donde la muestra está en condiciones de desarrollar el total de su potencial biolsgico como cada individuo lo requiera para que toda clla sea considerada propiamente laboral, y haya así correspondencia entre el número de habitantes $y$ el de brazos y mentes que en base a su estimación estamos considerando disponibles para participar en el esfuerzo que se requiera. Alcanzar esta meta significaría que a muy corto plazo estaríamos on condiciones de alimentar suficientemente a una población en crecimiento, y contribuit con un excedente a la alimentación de los pueblos que van quedando a la zaga; lo que vendría a redundarnos un más alto ingreso nacional que bien aprovechado contribuitá a una mayor expansión industrial, creación de nuevas fuentes de riquezas, y un cada vez más alto desarrollo socio-económico y bienestar individual. Prontamente pasaríamos a ocupar un lugar entre aquellos países que viven holgadamente, a pesar de estar mucho más densamente poblados, porque gracias a su mayor desarrollo disponen de mejor tecnología y suficientes recursos para industrializar y obtener altas utilidades por el destino y/o procesamiento que dan a la materia prima que poseen, o reciben de aquellos otros que por sus insuficientes recursos y tecnología deben entregarla en bruto, o a lo más semielaborada, con muy magras gananciales. Nos alínearíamos al fin entre los países bien desarrollados; pero siempre preocupados porque nositros ni otras naciones incrementen 
su población hasta el punto de bacer peligrar el necesario equilibrio entre población y recursos del mundo, para que una vida humana digna continúe siendo posible en esta tierra. La difecencia estaria en que con tal nuevo status permaneceríamos en el platillo más generoso de la balanza, y seguramente más dispuestos a entregar una cuota de nuestro esfuerzo para no sufrir las desastrosas consecuencias de un final desequilibrio; de las que en este evento no escapará ningún país. Ante esta alternativa la decisión es obvia.

En nuestro país los altos índices de desnutricion intrauterina, de malformaciones, de disfunciones y de otros sindromes deficitarios que se observan en nuestras Maternidades y la alta morbilidad que acontece durante el proceso del parto y en los primeros días de vida, todos ellos con elevada probabilidad invalidante en algún grado, exigen mucha aprehensión en una política de mejor aprovechamiento de los recursos humanos. Si a ello se suma el reconocido alto índice de desnutrición crónicamente mantenida, y la de los primeros meses de vida cuya influencia determinante de subdesarrollo bjológico, de mayor envergadura aún si acontece sobre un ser que nació con una desnutrición intrauterina, está suficientemente demostrado, tenemos que aceptar que un buen porcentaje de los niños que nacen año a año terminan por tener alguna incapacidad que en alguna medida les va a impedir desarrollar en plenitud su potencial genético. Como están influyendo los porcentajes acumulativos de estos daños y cuál es el futuro que nos depara en el rendimiento de la población escolar y laboral para una política de racionalización de recursos es una realidad no suficientemente magnificada, pero que urge conocer y determinar. Este conocimiento y la cuantificación de la población útil que debe orientar su actividad más a suplir estos deterioros que a una ocupación propiamente productiva, aparece indispensable cuando tengamos que defínir una política de salud interesada realmente en alinearnos entre dos países socio-económicamente ya desarrollados, pues ello invohtcra la necesidad de aumentar la población laboral propiamente tal, y si a la total nuestra le restamos aquélla con daños biologicos irrecuperables bien puede resultar que a lo mejor ella está disminuyendo. Si estábamos sobrepasados y así aparece conveniente tanto mejor aún, pero la mesura en la expansión de los programas masivos e indiscriminados de control de la fecundidad se hace en nuestro país por el momento aconsejable, basta no determinar exactamente cuánta parte de la nuestra está al servicio de la producción, y cuáles son las necesidades de recursos humanos para adentrarnos en el esfuerzo de un mayor desarrollo socio-económico. Esto no quiere decir que tengan que suprimirse o restringirsc los actuales programas de control sino sólo darle el carácter de instrumento al servicio de la familia, en un contexto con otras acciones de salud que centren su atención en prevenir la problemática mórbida expuesta y así propender en su conjunto al bienestar familiar; sobre todo cuando los recursos materiales y humanos de que disponemos se hacen insuficientes para solucionar todos los problemas de salud y superar el subdesaIrollo biológico.

Como las políticas de salud deben encausarse a solucionar los problemas de mayor relevancia no cabe duda que la primera jerarquía, en las acciones de salud que se programen, corresponde a todas aquelias que apuntan a prevenir estos mayores riesgos a que la madre y el niño están sometidos, y los derivados de déficits nutricionales. El objetivo debe ser incrementar la población con seres biológicamente bien desarrollados, capaces de participar con todo su potencial genético en el esfuerzo que debemos realizar para superar el subdesarrollo socio-económico, y aumentar la espectativa de vida de la población. Además de los programas de salud materna e infantil que cubran estos riesgos y otros de la mujer y del niño apare$\leftrightarrow$ indispensable cumplir otras actividades que propendan al mayor bienestar de la familia. Se configura así la necesidad de una "Planificación Familiar" que centre su preocupación para que las parejas puedan procrear el número de hijos que voluntaria $\mathrm{y}$ conscientemente decidan a fin dc constituir un hogar feliz, facilitándoles los medios que en un concepto de paternidad responsable soliciten para regular su fecundidad, o aquellas parejas esterriles para tener el hijo deseado; lo que implica programar además actividades educativas en la problemática de fertilidad y esterilidad. La regulación de fecundidad que se ofrezca, para limitar el número de hijos o espaciarlos entre ellos, debe ser con una metódica anticonceptiva científica y ética en la que el aborto, o cualquiera otra forma de inducirlo tiene que quedar absolutamente proscrito y considerado como un medio no lícito. Una "Planificación familiar" con tal intencionalidad surge nítidamente como una de las tantas actividades interesadas y tendientes al logro del bienestar de la familia, y es por tanto complementaria de todas las acciones de salud materna, infantil y otras de desarrollo social que se programen tras esa meta; siempre que como bienestar se entienda no sólo una preocupación por proteger o recuperar los riesgos de enfermar y morir de la madre y el niño, sino además y muy fundamentalmente porque las familias tengan capacidad autosuficiente para integrar sus miembros a la comunidad, una vez cumplidas las etapas formativas, con todo el potencial biológico e intelectual que logren desarrollarles con sus propios recursos, y los que eventualmente el Estado pudiera proporcionarles. Ello exige un equilibrio tal entre las con- 
diciones biológicas, psicoemocionales, económicas y culturales del núcleo familiar y su tamaño que les permita sobrellevar su propia existencia con dignidad y en permanente superación. Tanto los deterioros de salud como la procreación indiscriminada, no responsable ni autosuficiente, son factores que se confabulan para frenar el camino hacia el bienestar.

Si proyectamos esta concepción al plano nacional comprenderemos que la familia chilcna debe también orientarse con similares objetivos, aún cuando su crecimiento demográfico no sea un problema de especial consideración, dado que por iguales razones la influencia negativa de estos factores condicionales constituyc un serio impedimento para su progreso socjo-económico y bjenestar nacional.

Estos son los fundamentos básicos que obligan a promover la superación de los Servicios de Salud Materno-Infantiles y de Planificación Familiar en un conjunto de actividades del sector salud e intersectoriales.

Cualquiera sea la definición conceptual y la política que se acepte para el control del incremento poblacional no cabe duda que debe propenderse a entregar a la Sociedad individuos no dañados, para que así toda su población tenga la capacidad laboral propia de comunidades integradas con seres biológicamente bien desarrollados. Mantenernos en la situación actual es estrcchar cl círculo vicioso inteferente: subdesarrollo bioló gico subdesarrollo socio-cconómico, que fat tulmente nos conducirá a la ruina como nación. Un solo criteria tanto operativo como directivo en las acciones de fomento, protección y recuperación de la salud de la madre y el niño, sin afán parcelário ni hegemónico, surge como una imperiosa necesidad si se quicre evitar las disenciones que inevitabiemente acontecen cuando diferentes criterios deciden en la concepción del sujeto, o cn las acciones que apuntan a un mismo objetivo. No es audacia ascverar que el deterioro actual en gran parte se debe a que los programas siempre adolecicron de este defecto. La notoria desproporción en la disminución de la mortalidad neonatél con respecto a la infantil, ocurrida en esta última década, ponc en evidencia que el sujeto madrehijo no ha merecido la atención como tal y que las actividades individuaimente abordadas no han tenido igual trato.

Todas estas consideraciones las hago justamente porque la actividad regulación de la fecundidad, que es una de las tantas que abarca la problematica de la salud materno-infantil, está aflorando en el plano nacional como una panacea que permitirá solucionar los graves problemas de salud materna, infantil y de subsistencias, $y$ consecuencialmente erradicar la marginalidad. Variadas publisuciones pregonan que el control de la fecundidad ocupa uno de los lugares más prioritarios en políticas de salud, en circunstancia que sólo para nuestro desárrollo socio-económico su nccesidad no está bicn definida. Todo el deterioro en salud materno-in. fantil de uuestro país, que se evidencia en tan elevadas tasas de mortalidad materna, por aborto, infantil, nconatal y perinatul, y los altos índices, ni siquiera bicn cuantificados, de morbilidad, de desnutrición y de secuclas invalidantes, aparec: minimizado porque se piensa que sólo es consecuencia de un exceso de fecundidad, y no principalmente de esa desátención a que crónicanenta cste grupo etario ha estado sometido. Y lo mís trágico es que las más de las veces se le confunde como una sinonimia de "Planificación Familiar", que es justamente una actividad que apunta a todas las acciones que se involucran en un programa de salud Materno-Intantil bien concebido que a la regulación de la fecundidad en sí. Considerar este deterioros se hace indispensable pafa que entre los programas de salud Materna, Infantil y de Planificación Familiar se distribuyan equitativamente los presupuestos para todas las acciones conducentes a la protección de la familia, si realmente ésta es la neta. Las trasgresiones sólo traen reticencias y despiertan suspicacias, sobre todo cuando no se es consecuente con lo que se proclama como objetiro.

Una mejor organizáción y funcionamiento de las Matternidades y Hospitales Pediátricos para una atención oportuna y eficiente de la embarazada. del proceso del parto, del recién nacido, del nin̄o y de la madre en sus otros periodos, una princ:pal preocupación por la promoción de la consulta externa tanto de la patología como de los controles periódicos del riño y de la mujer embarazada. una programación adccuada para educar en salud y propender al saneamiento ambiental, evitar ef aborto, fomentar la alimentación natural y la paternidad responsable, prevenir lis enfermedades venéreas y el cáncer cérvico-utcrino, dar alimentación adecuada al niño, a la embarazada y a In madre en período de lactancia, solucionar los pro.. blemas de esterilidad de las parejas, y cn fin toclo un conjunto de acciones que bien coordinadas con los programas de regulación de la fecundidad propenden al bienestar de la familia, constituyen un todo insepariable que debe ser considerado en una politica de ":Salud Materno-Infantil" y de "Planificación Familiar" realmente interesada en la protección de la familia. Abocarse sólo a la regulación de la fecundidad, o siquiera darle una mayor jerarquía, es un error que no pueden cometer, aceptar ni dirigir quienes han vivido intensamente los problemas clínicos y conocen la problemática mórbida de la madre y el niño. A éstos corresponde participar decididamente para que una "Planificación Familiar", concebida en lá real cxtensión de 
su significado, se imponga sobre una acción de regulación de la fecundidad realizada aisladamente y desconexa de un programa materno-infantil integrado en todas sus acciones y objetivos. El rol del pediatra es de tanta trascendencia en el cumplimiento de estas acciones que debe incorporarse a estos Programas, tanto en los niveles directivos cormo en los de ejecución, sin ninguna reserva.

En Ia Política Nacional de Salud que ha comunicado el actual Gobierno, la atención a la madre y al niño aparece con carácter prioritario y orientado a la solución de los graves deterioros del área materno-infantil. Entre las actividades que se señalan figura la "Planificación Familiar" con una principal preocupación por el bienestar fami- liar y la educación sobre problemas de fecundidad. El control de ella como actividad independiente no se menciona y si en alguna declaración oficjal ha sido así manifestada por algunos expositores, seguramente ha sido porque estaban convencidos que quienes escuchaban o interpretaban, estando ya en conocimiento de la política declarada, la iban a considerar expresada como una sinonimia de "Planificación Familiar", programada en este contexto de acciones de Salud Materno-Infantiles expuesto. La consecución y augurosos resultados de esta política es la responsabilidad que como pediatras debemos asumir y encausar hacia una meta de bienestar familiar y superación socio-econórnica. 